Pacific Journal of Mathematics

A GENERALIZATION OF THE GLEASON-KAHANE-ŻELAZKO CHANG P'AO Ch'ÊN 


\section{A GENERALIZATION OF THE GLEASON-KAHANE-ZELAZKO THEOREM}

\section{Chang-Pao Chen}

In this paper, we consider two classes of commutative Banach algebras, which include $C^{n}(T), \operatorname{Lip}_{\alpha}(T), B V(T), L^{1} \cap L^{p}(G), A^{p}(G)$, $L^{1} \cap C_{0}(G), l^{p}, c_{0}$, and $C_{0}(S)$. We characterize ideals of finite codimension in these two classes of algebras and thereby partially answer a question suggested by $C$. $R$. Warner and $R$. Whitley.

In [5] and [9], A. M. Gleason, J. P. Kahane and W. Zelazko gave independently the following characterization of maximal ideals: Let $A$ be a commutative Banach algebra with unit element. Then a linear subspace $M$ of codimension 1 in $A$ is a maximal ideal in $A$ if and only if it consists of noninvertible elements, or equivalently, each element of $M$ belongs to some maximal ideal. This interesting result as first proved depended on the Hadamard Factorization Theorem.

This characterization of maximal ideals was extended in [15] and [16] to algebras without identity. In [16], C. R. Warner and R. Whitley also gave a characterization of ideals of finite codimension in $L^{1}(R)$ and $C[0,1]$. They showed: Let $A$ be any one of $L^{1}(R)$ and $C(S)$, where $S$ is a compact subset of $R$. If $M$ is a closed subspace of codimension $n$ in $A$ with the property that each element in $M$ belongs to at least $n$ regular maximal ideals, then $M$ is an ideal. In fact, $M$ is the intersection of $n$ regular maximal ideals. Also in [16], C. R. Warner and R. Whitley suggested the following question: For what locally compact abelian group $G$ does $L^{1}(G)$ have the property of $L^{1}(R)$ described above?

In this paper, we partially answer this question and generalize the work of C. R. Warner and R. Whitley. In this paper, two methods are introduced; One uses the Baire category theorem and the other generalizes the ideas of Theorems 2 and 4 in [16].

THEOREM 1. Let $A$ be a commutative Banach algebra with a countable maximal ideal space $\mathfrak{M}$. If $M$ is a closed subspace of codimension $n$ in $A$ with the property that each element in $M$ belongs to at least $n$ regular maximal ideals, then $M$ is an ideal, which is the intersection of $n$ regular maximal ideals. 
Proof. From the hypothesis, we know that $M \subset \cup I_{s_{1} s_{2}} \ldots s_{n}^{\prime}$ where $I_{s_{1} s_{2} \cdots s_{n}}$ denotes the space $\left\{x \in A: \hat{x}\right.$ vanishes at $\left.s_{1}, s_{2}, \ldots, s_{n}\right\}$ and the union is taken over all sets of distinct elements $s_{1}, s_{2}, \ldots, s_{n}$ in $\mathfrak{M}$. Since $\mathfrak{M}$ is countable, the union is a countable union. By the Baire category theorem, $M \subset I_{s_{1} s_{2} \cdots s_{n}}$ for some set of distinct elements $s_{1}, s_{2}, \ldots, s_{n}$ in $\mathfrak{M}$. If not, for any set of distinct elements $s_{1}, s_{2}, \ldots, s_{n}$ in $\mathfrak{M}$, we have $M \cap I_{s_{1} s_{2} \cdots s_{n}} \subsetneq M$. By the open mapping theorem, we find that $M \cap$ $I_{s_{1} s_{2} \cdots s_{n}}$ is of first category in $M$ and so the union $\cup\left(M \cap I_{s_{1} s_{2} \cdots s_{n}}\right)$ is of first category in $M$. This implies that $M$ is of first category in itself and contradicts the fact that $M$ is a Banach space. Therefore $M \subset I_{s_{1} s_{2} \cdots s_{n}}$ for some set of distinct elements $s_{1}, s_{2}, \ldots, s_{n}$ in $\mathfrak{M}$. Since $M$ and $I_{s_{1} s_{2}} \ldots s_{n}$ are of codimension $n$ in $A, M=I_{s_{1} s_{2} \cdots s_{n}}$. We have completed the proof.

EXAMPLE 2. Any of the following spaces has the property described in Theorem 1: $C^{n}(T) ; \operatorname{Lip}_{\alpha}(T), 0<\alpha \leq 1 ; B V(T) ; L^{p}(G), 1 \leq p \leq \infty$, or $A^{p}(G)$ or $C(G)$, or any normed ideal in $L^{1}(G)$, where $G$ is a metrizable compact abelian group; $l^{p}, 1 \leq p<\infty$, and $c_{0}$ (cf. $[1,2,4,7,8,10,11,12$, 14]).

REMARK 3. The structure of a metrizable compact abelian group can be found in [12, Theorem 2.2.6]. It is well-known that the maximal ideal space of $l^{\infty}$ coincides with the Stone-Čech compactification $\beta Z^{+}$, whose cardinal number is uncountable. (See [2, pp. 58] and [3, pp. 244].) Therefore Theorem 1 cannot be applied to this case. Theorem 1 answers the question suggested by C. R. Warner and R. Whitley for $L^{1}(G)$ in the case $G$ is compact and metrizable.

The following theorem extends the results presented in Theorem 1 to another kind of algebra while not hypothesizing that $M$ be closed. (Compare this with Theorem 1 and [16, Theorems 2 and 4].) This theorem generalizes Theorems 2 and 4 in [16].

THEOREM 4. Let $A$ be a commutative Banach algebra with involution $x \rightarrow x^{*}$ satisfying $\hat{x}^{*}=\hat{x}^{-}$. Suppose that there is an element $x_{0}$ in $A$, with $\hat{x}_{0}$ never zero, and that there is a one-to-one real-valued function $\phi$ on the maximal ideal space $\mathfrak{M}$ of $A$ such that $\hat{x}_{0} \phi^{j}=\hat{x}_{j}$ for some $x_{j}$ in $A$ $(1 \leq j \leq n)$. If $M$ is a subspace (not a priori closed) of codimension $n$ in $A$ with the property that each element in $M$ belongs to at least $n$ regular maximal ideals, then $M$ is an ideal which is the intersection of $n$ regular maximal ideals. 
Proof. Without loss of generality, we may assume that $\hat{x}_{0}$ is real-valued. Let $\bar{x}_{0}, \bar{x}_{1}, \ldots, \bar{x}_{n-1}$ denote the cosets in the quotient space $A / M$ corresponding to $x_{0}, x_{1}, \ldots, x_{n-1}$. If $\lambda_{0} \bar{x}_{0}+\lambda_{1} \bar{x}_{1}+\cdots+\lambda_{n-1} \bar{x}_{n-1}=\overline{0}$, then $\lambda_{0} x_{0}+\lambda_{1} x_{1}+\cdots+\lambda_{n-1} x_{n-1} \in M$ and so the equation $\lambda_{0}+$ $\lambda_{1} \phi(s)+\cdots+\lambda_{n-1} \phi(s)^{n-1}=0$ has $n$ distinct solutions in $s$. This implies that the polynomial $\lambda_{0}+\lambda_{1} t+\cdots+\lambda_{n-1} t^{n-1}$ has $n$ distinct zeros, which occurs only if all $\lambda$,'s are zero. Hence $\bar{x}_{0}, \bar{x}_{1}, \ldots, \bar{x}_{n-1}$ form a basis for $A / M$.

There exist scalars $\lambda_{0}, \ldots, \lambda_{n-1}$ such that $x_{n}-\lambda_{0} x_{0}-\cdots-\lambda_{n-1} x_{n-1}$ is in $M$. Denote this element of $M$ by $m_{0}$. We claim that $\hat{m}_{0}$ is real-valued. By hypothesis and since $m_{0} \in M$, we find that the equation $\lambda_{0}+\lambda_{1} \phi(s)$ $+\cdots+\lambda_{n-1} \phi(s)^{n-1}=\phi(s)^{n}$ has $n$ distinct solutions, say $s_{1}, s_{2}, \ldots, s_{n}$. We write down these relations as follows:

$$
\begin{gathered}
\lambda_{0}+\lambda_{1} \phi\left(s_{1}\right)+\cdots+\lambda_{n-1} \phi\left(s_{1}\right)^{n-1}=\phi\left(s_{1}\right)^{n}, \\
\vdots \\
\lambda_{0}+\lambda_{1} \phi\left(s_{n}\right)+\cdots+\lambda_{n-1} \phi\left(s_{n}\right)^{n-1}=\phi\left(s_{n}\right)^{n} .
\end{gathered}
$$

By hypothesis, we know that $\phi\left(s_{1}\right), \phi\left(s_{2}\right), \ldots, \phi\left(s_{n}\right)$ are $n$ distinct real numbers. By Cramer's rule, we find that $\lambda_{0}, \lambda_{1}, \ldots, \lambda_{n-1}$ are all real and so $\hat{m}_{0}$ is real-valued. As we saw above, $\hat{m}_{0}$ vanishes exactly at $s_{1}, s_{2}, \ldots, s_{n}$.

Let $m$ be an element in $M$ with $\hat{m}$ real-valued. We have $m+i m_{0} \in M$ and so the equation $\hat{m}(s)+i \hat{m}_{0}(s)=0$ has $n$ distinct solutions in $s$. This implies that $\hat{m}\left(s_{1}\right)=\cdots=\hat{m}\left(s_{n}\right)=0$, because $\hat{m}_{0}$ vanishes exactly at $s_{1}, s_{2}, \ldots, s_{n}$.

Fix $m$ in $M$. There exist scalars $\lambda_{0}, \lambda_{1}, \ldots, \lambda_{n-1}$ such that $m^{*}-\lambda_{0} x_{0}$ $-\cdots-\lambda_{n-1} x_{n-1}$ is in $M$. We have $m+m^{*}-\lambda_{0} x_{0}-\cdots-\lambda_{n-1} x_{n-1} \in$ $M$ and so the equation $2 \operatorname{Re} \hat{m}(s)-\lambda_{0} \hat{x}_{0}(s)-\cdots-\lambda_{n-1} \hat{x}_{0}(s) \phi(s)^{n-1}=$ 0 has $n$ distinct solutions in $s$. By Cramer's rule, we find that $\lambda_{0}, \lambda_{1}, \ldots, \lambda_{n-1}$ are all real. On the other hand, we have $-m+m^{*}-$ $\lambda_{0} x_{0}-\cdots-\lambda_{n-1} x_{n-1} \in M$ and so the equation $-2 i \operatorname{Im} \hat{m}(s)-$ $\lambda_{0} \hat{x}_{0}(s)-\cdots-\lambda_{n-1} \hat{x}_{0}(s) \phi(s)^{n-1}=0$ has $n$ distinct solutions in $s$. By Cramer's rule, we find that $\lambda_{0}, \lambda_{1}, \ldots, \lambda_{n-1}$ are all pure imaginary. Combining these two results we find that all $\lambda_{j}$ 's are zero. This shows that $m^{*}$ is in $M$.

We know that

$$
m=2^{-1}\left(m+m^{*}\right)+i\left[(2 i)^{-1}\left(m-m^{*}\right)\right]
$$


where the Fourier-Gelfand transforms of $m+m^{*}$ and $(2 i)^{-1}\left(m-m^{*}\right)$ are real-valued. From the results presented in the preceding two paragraphs, we find that $\hat{m}$ vanishes at $s_{1}, s_{2}, \ldots, s_{n}$ for every $m$ in $M$. This says that $M \subset I_{s_{1} s_{2} \cdots s_{n}}$, where $I_{s_{1} s_{2} \cdots s_{n}}$ denotes the space $\{x \in A: \hat{x}$ vanishes at $\left.s_{1}, s_{2}, \ldots, s_{n}\right\}$. Since $M$ and $I_{s_{1} s_{2} \cdots s_{n}}$ are of codimension $n$ in $A, M=I_{s_{1} s_{2} \cdots s_{n}}$. We have completed the proof.

EXAMPLE 5. Any of the following spaces has the property described in Theorem 4: $C^{n}(T) ; \operatorname{Lip}_{\alpha}(T), 0<\alpha \leq 1 ; B V(T) ; L^{1} \cap L^{p}(G), 1 \leq p \leq \infty$, or $A^{p}(G)$ or $L^{1} \cap C_{0}(G)$, or any normed ideal in $L^{1}(G)$ which is invariant under involution, where $G$ is either a metrizable compact abelian group or the direct product of the real line $R$ and a metrizable compact abelian group; $l^{p}, 1 \leq p<\infty$, and $C_{0}(S)$, where $S$ is any closed subset of $R \times Z^{\infty}$.

Example 5 follows immediately from the following lemma:

LEMMa 6. The following two types of algebras have the property described in Theorem 4:

(i) Any normed ideal in $L^{1}(G)$ which is invariant under involution, where $G$ is a metrizable compact abelian group or the direct product of $R$ and such $a G$.

(ii) $C_{0}(S)$, where $S$ is any closed subset of $R \times Z^{\infty}$.

Proof. Let $A$ be a normed ideal in $L^{1}(G)$ which is invariant under involution, where $G$ is either a metrizable compact abelian group or the direct product of the real line $R$ and a metrizable compact abelian group. From Theorems 2.2.2 and 2.2.6 in [12] we find that $\Gamma$ is of the form $\Gamma_{1} \times \Gamma_{2}$, where $\Gamma_{1}$ is $\{0\}$ or $R$ and $\Gamma_{2}$ is countable. Write $\Gamma_{2}$ as $\left\{\gamma_{1}, \gamma_{2}, \ldots\right\}$. Define a function $\phi$ on $\Gamma$ as follows:

$$
\begin{gathered}
\phi\left(\gamma_{m}\right)=m \text { if } \Gamma_{1}=\{0\}, \\
\phi\left(x, \gamma_{m}\right)=\frac{x}{\left(1+4 \pi^{2} x^{2}\right)^{1 / 2}}+m \text { if } \Gamma_{1}=R,
\end{gathered}
$$

then $\phi$ is a one-to-one real-valued function on $\Gamma$.

Choose an integrable function $h_{0}$ on $G$ with the following property:

$$
\begin{gathered}
\hat{h}_{0}\left(\gamma_{m}\right)=e^{-m^{2}} \quad \text { if } \Gamma_{1}=\{0\}, \\
\hat{h}_{0}\left(x, \gamma_{m}\right)=e^{-\left(x^{2}+m^{2}\right)} \quad \text { if } \Gamma_{1}=R .
\end{gathered}
$$


It is well-known that $\Gamma$ is sigma-compact, say $\Gamma=\cup_{j=1}^{\infty} K_{j}$, where $K_{j}$ are compact subsets of $\Gamma$. There exists functions $g_{j}$ in $A$ such that $\hat{g}_{j} \geq 0$ on $\Gamma$ and $\hat{g}_{j}=1$ on $K_{j}$. Define

$$
g_{0}=\sum_{j=1}^{\infty} \frac{g_{j}}{j^{2}\left\|g_{j}\right\|_{A}} \text { and } f_{0}=g_{0} * h_{0}
$$

then $f_{0}$ is in $A$ and $\hat{f}_{0}$ is never zero.

For the case $\Gamma_{1}=R$ we have

$$
\begin{aligned}
\hat{f}_{0}\left(x, \gamma_{m}\right) \phi\left(x, \gamma_{m}\right)^{j} & =\hat{g}_{0}\left(x, \gamma_{m}\right) e^{-\left(x^{2}+m^{2}\right)}\left[\frac{x}{\left(1+4 \pi^{2} x^{2}\right)^{1 / 2}}+m\right]^{j} \\
& =\hat{g}_{0}\left(x, \gamma_{m}\right) e^{-\left(x^{2}+m^{2}\right)} \sum_{k=0}^{j}\left(\begin{array}{l}
j \\
k
\end{array}\right) x^{k} \hat{G}_{1}(x)^{k} m^{j-k} \\
& =\hat{g}_{0}\left(x, \gamma_{m}\right) \sum_{k=0}^{j}\left(\begin{array}{l}
j \\
k
\end{array}\right) e^{-x^{2}} x^{k} \hat{G}_{1}(x)^{k} e^{-m^{2}} m^{j-k} \\
& =\hat{g}_{0}\left(x, \gamma_{m}\right) \sum_{k=0}^{j}\left(\begin{array}{l}
j \\
k
\end{array}\right) \hat{H}_{k}(x) \hat{G}_{1}(x)^{k} e^{-m^{2}} m^{j-k} \\
& =\hat{g}_{0}\left(x, \gamma_{m}\right) \hat{F}_{j}\left(x, \gamma_{m}\right) \\
& =\hat{f}_{j}\left(x, \gamma_{m}\right)
\end{aligned}
$$

where

$$
\begin{gathered}
\left(\begin{array}{l}
j \\
k
\end{array}\right)=\frac{j(j-1)(j-2) \cdots(j-k+1)}{k !}, \quad\left(\begin{array}{l}
j \\
0
\end{array}\right)=1, \\
G_{1}(x)=\frac{1}{(4 \pi)^{1 / 2}} \frac{1}{\Gamma(1 / 2)} \int_{0}^{\infty} e^{-\pi x^{2} / \delta} e^{-\delta / 4 \pi} \frac{d \delta}{\delta}, \\
\hat{H}_{k}(x)=e^{-x^{2} x^{k}}, \\
F_{j}=\sum_{k=0}^{j}\left(\begin{array}{l}
j \\
k
\end{array}\right)(H_{k} * \underbrace{G_{1} * \cdots * G_{1}}_{k \text { terms }})\left(\sum_{m=1}^{\infty} e^{-m^{2}} m^{j-k} \gamma_{m}\right), \\
f_{J}=g_{0} * F_{j} .
\end{gathered}
$$

The definition of $G_{1}$ can be found in [13, pp. 132]. The existence of integrable functions $H_{k}$ on $R$ is based on the fact that the function $e^{-x^{2}}$ is 
rapidly decreasing. We have $G_{1} \in L^{1}(R), H_{k} \in L^{1}(R)$ and the functions

$$
\sum_{m=1}^{\infty} e^{-m^{2}} m^{j-k} \gamma_{m}
$$

are integrable. This implies that $F_{j} \in L^{1}(G)$ and so $f_{J}$ is in $A$. This result is also true for the case $\Gamma_{1}=\{0\}$; with minor modifications the preceding proof applies.

It remains to show (ii). Let $S$ be any closed subset of the space $R \times Z^{\infty}$. From Theorem XI.6.5 in [3] we find that $S$ is locally compact. It is well-known that $R \times Z^{\infty}$ is the dual group of $R \times T^{\omega}$. (See [12, §2.2].) Take $G=R \times T^{\omega}$ and define $\phi$ and $h_{0}$ as above. Denote the restriction of $\hat{h}_{0}$ on $S$ by $f_{0}$ and the restriction of $\phi$ on $S$ by itself, then $f_{0} \in C_{0}(S), f_{0}$ is never zero, $\phi$ is one-to-one and real-valued and $f_{0} \phi^{\prime} \in C_{0}(S)$ for all $j$. (Here we use the assumption that $S$ is closed.) We have completed the proof.

The problem of characterizing the ideals of finite codimension for $L^{1}\left(R^{2}\right)$ and $C(D), D$ the closed unit disk, raised in [16] remains open.

Acknowledgement. I would like to thank the referee for his valuable suggestions.

\section{REFERENCES}

[1] J. Cigler, Normed ideals in $L^{1}(G)$, Nederl. Akad. Wetensch. Indag. Math., 31 (1969), 273-282.

[2] R. G. Douglas, Banach Algebra Techniques in Operator Theory, Academic Press, New York and London, 1972.

[3] J. Dugundji, Topology, Allyn and Bacon, Boston 1966.

[4] R. E. Edwards, Fourier Series, a Modern Introduction, 2 Vols. New York, N. Y.: Holt, Rinehart and Winston, Inc. 1967.

[5] A. M. Gleason, A characterization of maximal ideals, J. Analyse Math., 19 (1967), $171-172$.

[6] E. Hewitt and K. A. Ross, Abstract Harmonic Analysis, Vol. I, Springer-Verlag, Berlin, 1963.

[7] _ Abstract Harmonic Analysis, Vol. II, Springer-Verlag, Berlin, 1970.

[8] E. Hewitt and K. Stromberg, Real and Abstract Analysis, Springer-Verlag, New York, 1965.

[9] J. P. Kahane and W. Zelazko, A characterization of maximal ideals in commutative Banach algebras, Studia Math., 29 (1968), 339-343.

[10] Y. Katznelson, An Introduction to Harmonic Analysis, New York, 1968.

[11] H. Reiter, Classical Harmonic Analysis and Locally Compact Groups, Oxford, 1968.

[12] W. Rudin, Fourier Analysis on Groups, Interscience, New York, 1962.

[13] E. M. Stein, Singular Integrals and Differentiability Properties of Functions, Princeton, 1970. 
[14] H. C. Wang, Homogeneous Banach Algebras, Lecture Notes in Pure and Appl. Mathematics, Dekker, New York, 1977.

[15] C. R. Warner and R. Whitley, A characterization of regular maximal ideals, Pacific J. Math., 30 (1969), 277-281.

[16] Ideals of finite codimension in $C[0,1]$ and $L^{1}(R)$, Proc. Amer. Math. Soc., 76 (1979), 263-267.

Received September 10, 1981 and in revised form January 20, 1982.

INSTITUTE OF MATHEMATICS

National TSING HUa University

HSINCHU 300, TAIWAN

REPUBLIC OF CHINA 



\section{PACIFIC JOURNAL OF MATHEMATICS}

\section{EDITORS}

Donald BabbitT (Managing Editor)

University of California

Los Angeles, CA 90024

Hugo Rossi

University of Utah

Salt Lake City, UT 84112

C. C. Moore and Arthur Ogus

University of California

Berkeley, CA 94720
J. DugunduI

Department of Mathematics

University of Southern California

Los Angeles, CA 90089-1113

R. FinN and H. SAmelson

Stanford University

Stanford, CA 94305

\section{ASSOCIATE EDITORS}
R. ARENS
E. F. BECKENBACH
B. H. NEUMANN
F. WOLF
K. YosHIDA (1906-1982)

\section{SUPPORTING INSTITUTIONS}

UNIVERSITY OF ARIZONA

UNIVERSITY OF BRITISH COLUMBIA

CALIFORNIA INSTITUTE OF TECHNOLOGY

UNIVERSITY OF CALIFORNIA

MONTANA STATE UNIVERSITY

UNIVERSITY OF NEVADA, RENO

NEW MEXICO STATE UNIVERSITY

OREGON STATE UNIVERSITY
UNIVERSITY OF OREGON

UNIVERSITY OF SOUTHERN CALIFORNIA

STANFORD UNIVERSITY

UNIVERSITY OF HAWAII

UNIVERSITY OF TOKYO

UNIVERSITY OF UTAH

WASHINGTON STATE UNIVERSITY

UNIVERSITY OF WASHINGTON 


\section{Pacific Journal of Mathematics}

Vol. 107, No. $1 \quad$ January, 1983

John Kelly Beem and Phillip E. Parker, Klein-Gordon solvability and the

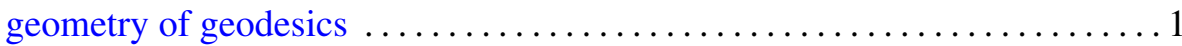

David Borwein and Amnon Jakimovski, Transformations of certain

sequences of random variables by generalized Hausdorff matrices ..... 15

Willy Brandal and Erol Barbut, Localizations of torsion theories . . . . . . . 227

John David Brillhart, Paul Erdős and Richard Patrick Morton, On sums

of Rudin-Shapiro coefficients. II ........................... 39

Martin Lloyd Brown, A note on tamely ramified extensions of rings $\ldots \ldots \ldots 71$

Chang P'ao Ch'ên, A generalization of the Gleason-Kahane-Żelazko

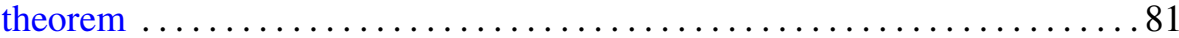

I. P. de Guzman, Annihilator alternative algebras $\ldots \ldots \ldots \ldots \ldots \ldots$. . . . 89

Ralph Jay De Laubenfels, Extensions of $d / d x$ that generate uniformly

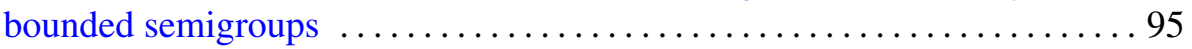

Patrick Ronald Halpin, Some Poincaré series related to identities of $2 \times 2$

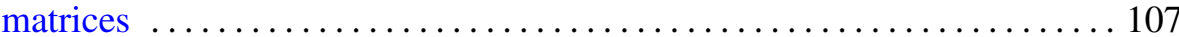

Fumio Hiai, Masanori Ohya and Makoto Tsukada, Sufficiency and

relative entropy in $*$-algebras with applications in quantum systems . . . 117

Dean Robert Hickerson, Splittings of finite groups $\ldots \ldots \ldots \ldots \ldots \ldots \ldots 14$

Jon Lee Johnson, Integral closure and generalized transforms in graded

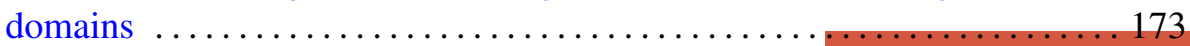

Maria Grazia Marinari, Francesco Odetti and Mario Raimondo, Affine

curves over an algebraically nonclosed field . ................. 179

Douglas Shelby Meadows, Explicit PL self-knottings and the structure of

PL homotopy complex projective spaces $\ldots \ldots \ldots \ldots \ldots \ldots \ldots \ldots \ldots$

Charles Kimbrough Megibben, III, Crawley's problem on the unique

$\omega$-elongation of $p$-groups is undecidable .................... 205

Mary Elizabeth Schaps, Versal determinantal deformations $\ldots \ldots \ldots \ldots 213$

Stephen Scheinberg, Gauthier's localization theorem on meromorphic

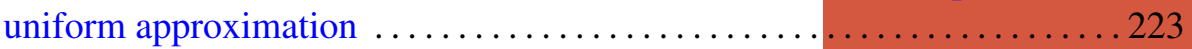

Peter Frederick Stiller, On the uniformization of certain curves . . . . . . . 229

Ernest Lester Stitzinger, Engel's theorem for a class of algebras . . . . . . . . 245

Emery Thomas, On the zeta function for function fields over $F_{p} \ldots \ldots \ldots 251$ 